\title{
Application of Cloud Computing Technology and Big Data Analysis in Electronic Commerce
}

\author{
Xue Bai \\ Department of Economic management, Guangdong Polytechnic, Foshan, China \\ chenxiaoyunld@163.com
}

Keywords: Cloud computing; Big data; Electronic commerce; Search engines; Enterprise

\begin{abstract}
Cloud computing is the ability to use high-speed Internet transmission, the data processing process from a personal computer or enterprise server to the cloud. Cloud computing provides a more powerful search engine for e-commerce. Electronic commerce model based on cloud computing improves the core competitiveness of enterprises. This paper analyzes and explores the main effects and functions of cloud computing and big data processing technology in the development of electronic commerce. In this paper, the application of cloud computing technology and big data analysis in electronic commerce is proposed.
\end{abstract}

\section{Introduction}

Electronic commerce is a new type of economic activity, which is gradually developed through the integration of market and information technology and the deepening of social specialization. Generally speaking, the concept of electronic commerce to from generalized and narrow sense distinction, general e-commerce is that contains all the business activities of the electronic technology, the narrow sense of e-commerce that rely on the computer network as the basis for online transactions of business activities.

Big data refers to the amount of information needed is too large, has been more than a computer in the processing of data can be used by the amount of memory. The general concept is that in a certain period of time through the traditional technology can not be achieved, management, processing of the data set of the general term [1]. Reilly media company of large data definition is more focused on big data management and analysis system in the key role that when data capacity and performance to large enough to be the implementation of the data management and analysis system for design and decision factors, can be referred to as data. Currently big data does not have an authoritative definition, different enterprises, institutions, experts on the definition of big data is not the same. Overall, for the not the same definition there are also similarities, namely data not only a large number and diverse forms, complex structure and traditional techniques is difficult to meet the requirements of real-time data processing.

Cloud computing technology is divided into four key steps: hardware, cloud operating system, cloud platform software and cloud application software. Cloud computing is the use of the transmission capacity of high-speed Internet, data processing from a personal computer or server enterprise transfer to the cloud, enterprise and individual users no need to input the expensive hardware acquisition cost, only need through the Internet to lease or buy the super meter count machine cluster service super computing functions [2]. Cloud computing e-commerce model is to improve the core competitiveness of enterprises.

Big data era, data has become the most valuable assets of enterprises. The economic benefit of data has been widely used, the development direction of the enterprise, strategic decision, target structure, value analysis and the potential of mining and data are closely related. Enterprise future development and competition, will be the scale of data development and competition. The storage, pricing, management and driving of data assets will be an innovative business model. Big data in the future will 
become the basic conditions constitute the elements of the enterprise. In this paper, the application of cloud computing technology and big data analysis in electronic commerce is proposed.

\section{The Influence of Cloud Computing Technology to the Development of Electronic Commerce}

Cloud computing can provide a reliable and economical system for electronic commerce system. The software is a kind of service which is provided by SaaS. It provides the software as an online service. Electronic outsourcing based on cloud computing technology is one of the important applications of enterprise application e-commerce services.

Enterprise in the use of network architecture and application of cloud computing can make it more convenient, electronic outsourcing is actually a form of electronic commerce with the need to change. Enterprise in the use of cloud computing services, for the development and upgrading of e-commerce system has no longer need to spend a lot of money and manpower, do not need to invest in the establishment of a separate internal trap software and procedures. As a client of the enterprise can be more convenient to use cloud computing provides a variety of services, then only need to install a web browser can be, which makes the enterprise for the maintenance and upgrade of electronic commerce system and input costs more. From this, we can know that the cloud computing to improve the flexibility and professionalism of enterprise e-commerce applications, as is shown by equation (1) [3].

$$
W_{2 j} f(n)=2^{-j / 2} \int_{-\infty}^{\infty} f(x) \overline{\psi\left(2^{-j} x-k\right)} d x
$$

Cloud computing can greatly reduce the cost of enterprise electronic commerce application cloud computing to the computing power as a "commodity" were in circulation, the commodity "through the Internet transmission and shared among multiple users, unlimited and expansion, which makes resource utilization rate in order to improve.

On the one hand, cloud computing application in the electronic commerce to reduce the cost of enterprise electronic commerce system established; on the other hand, cloud computing to focus all of the computing resources and software realization of automatic management, without human involvement [4]. The cloud computing service providers without tedious details and trouble, to focus more on their own business, is conducive to innovation and reduce costs to cloud computing provide computing service to users over the Internet, will form scale economy effect.

Cloud computing has beyond the imagination of computing power, even small and micro enterprises are able to have the information processing capabilities and speed comparable to the type of multinational enterprises. Electronic business web site through a variety of means to increase website browsing and sales volume, such as double eleven, website traffic will erupt type grows, if there is no sufficient technical preparation, the server and to many users logged on simultaneously will appear when the jam or even collapse phenomenon in the. However, through the cloud computing server cluster technology, with the cloud database storage, you can automatically allocate enough for the enterprise to meet the needs of the enterprise server quantity and data access.

Cloud computing as a new computer technology, with high efficiency, low cost, high safety, convenient services, the sharing of resources advantages, as is shown by equation(2), where c is to make up for the deficiency of the traditional electronic commerce, the electronic commerce service efficiency higher, lower cost, more reliable, stable and convenient.

$$
C_{1}=\frac{1}{\sum_{i=1}^{n} k\left(\left\|\frac{y_{0}-\mathrm{X}_{i}}{h_{1}}\right\|^{2}\right)\left(1+d\left(\mathrm{X}_{i}\right)\right)}
$$

Cloud computing with virtualization technology, will be distributed in different areas of computing resources to integrate, to achieve the sharing of basic resources. Electronic commerce enterprises can 
use the cloud computing to provide the powerful ability to work together to achieve the sharing of information resources [5]. Cloud computing can also be based on the needs of e-commerce business expansion, will be more than one commodity information resources sharing, reduce the construction of independent e-commerce sites, the funds and time.

Cloud computing is an effective way to ensure the security and reliability of electronic commerce, so as to ensure the distributed implementation of the calculation, so that the software can be used as a time service to each user. In the future, you can get a cloud computing system can effectively achieve without the virus and the safety of the data, with storage and sharing more convenient and network services to expand the computing resources, and many other advantages.

\section{Analysis on the Application of Big Data Analysis in Electronic Commerce Service}

Big data with $4 \mathrm{~V}$ features, including Volume (a large number), Velocity (high speed), Variety (diversity), and Value. With the advent of the era of cloud, big data has become the industry's most popular darling, but the strategic significance of big data is based on the integration of data information, analysis, processing, and in order to bring benefits.

As the electronic commerce and the vast amounts of data for real-time analysis, has become the current e-commerce competition the main focus, and the main value of the data is detailed analysis and use of the relevant information [6]. Using cloud computing can be in a relatively short period of time for more data for collection, storage, analysis and processing, thus greatly enhances the enterprise data processing and information analysis ability, making electricity supplier to real-time accurate mining related data, and in-depth analysis of the data, as is shown by equation (3).

$$
\tilde{g}(n)=(-1)^{n-1} h(-n+1) \quad g(n)=(-1)^{n-1} \tilde{h}(-n+1)
$$

The introduction of the Internet by the user generated data model. The model provides low cost, strong timeliness of information. And the authenticity and reliability of the data need to be verified. E-commerce and traditional sales methods have a big advantage, that is, the availability of data. In the traditional sales, enterprises are rarely able to understand the source of customers to visit, if now do not buy the future will not buy in, and electronic commerce can understand to customers to buy, used behavior, through these data help the enterprise to sell their products, thus better for the customer to provide services.

In various commercial departments in the formation of the information of the blocking state, it is a fundamental constraint on the wide range of data applications [7]. Although in the process of data, the technologies have been continuously innovation, and fundamentally improve the processing capacity of the data. But due to the source of large data channels varied, more complex, especially through a variety of channels to get the data, and some may not use the data. These no use of the data in a certain extent will interfere with the analysis of the results of the data. And the use of the data and the actual situation there is a considerable distance, as is shown by equation (4).

$$
E\left[\tilde{X} \tilde{X}^{T}\right]=\left(H^{T} U H\right)^{-1} H^{T} U R U H\left(H^{T} U H\right)^{-1}
$$

At the same time, on the other hand, it is able to carry out the information push service, as well as the hot spot information to recommend and push the new information retrieval service. The related work in most cases is in a specific field, and is based on static network environment and build the corresponding network database system, the in a great extent can not use the uncertainty of the current network environment database resources [8]. It is essential that you will set up a database grid system for multi domain dynamic data integration, can effectively for resource database management. The accuracy and to be able to improve access to resources, and it is improve the efficiency of the corresponding query, the database resource until the good management effect. 
Therefore, the source of the data channel is more extensive, the analysis of the data to be timely and so on is also increasingly presented [9]. The era of big data and the Internet, is a major change in the field of information technology, and has become an important new strategic resource, by the high attention of the government of all countries. Countries through a large number of complex data for further collection and collation, not only to improve the ability to predict the direction of social development, but also conducive to create a new business model in all fields.

\section{Research on Application of Cloud Computing Technology and Big Data Analysis in Electronic Commerce}

The creation of the value of electronic commerce mainly from four aspects: the level of efficiency, whether complementary, lock and innovation functions, etc.. Efficiency mainly refers to the electronic commerce information with fast and efficient information transfer function. Complementary effect mainly refers to the information of the exchange of the two sides complement each other, in order to form a certain economic effect. Lock function is to fully meet the needs of customers, to lock a large number of customer groups, to form a stable customer base. Function innovation is refers to the continuous innovation of products and services, especially under the background of China's big data, e-business value creation is gradually showing the new changes and new change in order to adapt to the market a wide range of requirements.

Cloud computing on the impact of the search engine is generally as follows: to provide a powerful web search service. With the rapid development of the Internet, there are hundreds of millions of Chinese web pages in the world [10]. Then such a huge number of pages, and each page at least a few hundred K capacity, existing search engine has been difficult to deal with, not to mention these web information real time in dynamic status updates, even is each one second updated, for example, such sites may in just a few seconds to climb a. Thus, to provide users with efficient, high-quality search service will be the key technology to promote the development of electronic commerce.

To provide enterprise remote data backup and disaster recovery services. With the increasing amount of data in the electronic commerce enterprise, the security of data is also increasing. The electronic commerce enterprise data not only to have sufficient capacity to store, also need to implement data backup and remote disaster recovery. Not only to ensure the safety of local data, but also it is to ensure that when a major disaster occurring locally, and it is by remote backup or remote disaster recovery system for fast recovery.

Data security issues more prominent, with the development of new technologies, more convenient to collect and collate data, so through the collection of various channels, in order to collect the comprehensive information of consumers. Consumer information mainly includes: basic information, information, social information and location information in the transaction layer. While providing personalized service for consumers, but also to the maximum extent to ensure the security of information, to protect the user's privacy, as shown in Fig. 1.

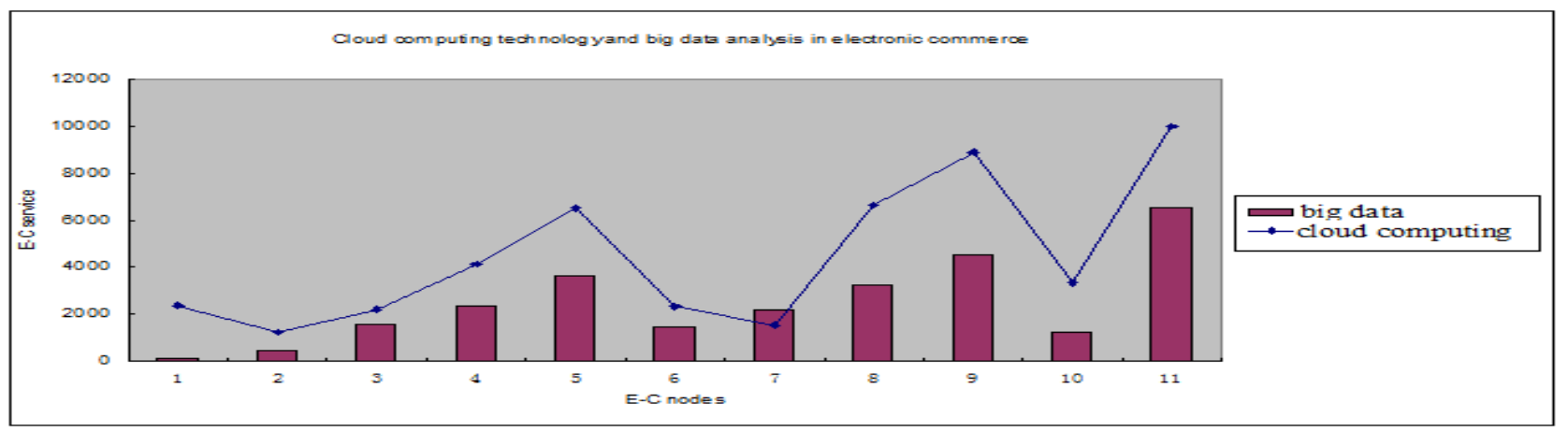

Figure 1. Application of cloud computing technology and big data analysis in electronic commerce 
Big data market size is explosive growth, the formation of large industrial chain, which has produced a data service model of e-commerce services. In the era of big data, the data scale competition is particularly fierce; all commercial enterprises want to through the data information fully optimize all aspects of the enterprise operation. However, in fact the majority of enterprises no such funds or technology for large data of optimal allocation to arm themselves, under the background of such demand outlook, those with advantages in capital and technology allows the electronic commerce enterprise massive data resources to carry on the computation and analysis, form data information product marketing to small and medium-sized enterprises. Thus establishes the new electronic commerce service pattern.

\section{Summary}

Cloud computing in the big data environment for the integration of e-commerce, and it is especially for the collection of information, information analysis, and storage and security requirements. Society of our country economy is gradually to the era of big data forward, a lot of information to the economic life influx, how the effective use of the information and data processing technology of modern electronic business and trade activities to carry out comprehensive development is an important topic in the present economic level of concern and study. In this paper, the application of cloud computing technology and big data analysis in electronic commerce is proposed. The outbreak and application of large data is changing the traditional business methods, cloud computing has opened up a new area of development for e-commerce.

\section{Acknowledgements}

This paper is supported by Guangdong province colleges and universities outstanding young teacher training project (Yq2013172).

\section{References}

[1] Asgarali Bouyer, "Resource Discovery and Selection Service based on Optimized Inflation in Market-oriented Cloud Computing Environment", JCIT, Vol. 9, No. 3, pp. 76 85, 2014.

[2] Dawei Sun, Ge Fu, Xinran Liu, Hong Zhang, "Optimizing Data Stream Graph for Big Data Stream Computing in Cloud Datacenter Environments", IJACT, Vol. 6, No. 5, pp. 53 65, 2014.

[3] S.Balasubramaniam, V.Kavitha, "A Survey on Data Retrieval Techniques in Cloud Computing", JCIT, Vol. 8, No. 16, pp. $15 \sim 24,2013$.

[4] N.R.RamMohan, E.Baburaj, "Resource Allocation Using Interference Aware Technique in Cloud Computing Environment", JDCTA, Vol. 8, No. 1, pp. $35 \sim$ 46, 2014.

[5] Hyoung woo Park, Il Yeon Yeo, Haengjin Jang, Seo-Young Noh, "Simulation based Analysis on Big Data Service Bottleneck for Data Center", JNIT, Vol. 4, No. 8, pp. 185 189, 2013.

[6] Tao Huang, Zhiyong Zhang, Qingli Chen, Yanan Chang, "A Method for Trusted Usage Control over Digital Contents Based on Cloud Computing", JDCTA, Vol. 7, No. 4, pp. 795 802, 2013.

[7] Du Jin, "Application of "Internet of Things" in Electronic Commerce", JDCTA, Vol. 6, No. 8, pp. $222 \sim 230,2012$.

[8] Md. Motaharul Islam, Pham Phuoc Hung, Al-Amin Hossain, Mohammad Aazam, "A Framework of Smart Internet of Things based Cloud Computing", RNIS, Volume 14, pp. 646 651, 2013. 
[9] Sirivimol Thanchalatudom, Namfon Assawamekin, "Using Big Data Technology for Information Management in Hybrid Learning System", RNIS, Volume 12, pp. 179 182, 2013.

[10] Hongxin Wan, Yun Peng, "Clustering and Evaluation on Electronic Commerce Customers Based on Fuzzy Set", IJACT, Vol. 5, No. 3, pp. 199 206, 2013. 